\title{
Upregulated expression and activation of membrane-associated proteases in esophageal squamous cell carcinoma
}

\author{
KATARZYNA AUGOFF $^{1}$, ANITA HRYNIEWICZ-JANKOWSKA ${ }^{2}$, RENATA TABOLA ${ }^{1}$, \\ LESZEK CZAPLA $^{1}$, PIOTR SZELACHOWSKI ${ }^{1}$, JAROSLAW WIERZBICKI $^{3}$, \\ KRZYSZTOF GRABOWSKI ${ }^{1}$ and ALEKSANDER F. SIKORSKI ${ }^{2}$ \\ ${ }^{1}$ Department of Gastrointestinal and General Surgery, Wroclaw Medical University, Wroclaw; \\ ${ }^{2}$ Laboratory of Cytobiochemistry, Biotechnology Faculty, University of Wroclaw, Wroclaw; \\ ${ }^{3}$ Department of Minimally Invasive Surgery and Proctology, Wroclaw Medical University, Wroclaw, Poland
}

Received February 25, 2014; Accepted April 2, 2014

DOI: $10.3892 /$ or.2014.3162

\begin{abstract}
To better understand the role of membraneassociated proteolytic systems in the development of esophageal cancer, we studied the expression of two serine proteases, fibroblast activation protein- $\alpha$ (FAP- $\alpha$ ) and dipeptidyl peptidase IV (DPPIV) and three metalloproteinases, matrix metalloproteinase (MMP)-2, MMP-9 and MT1-MMP in 24 primary esophageal squamous cell carcinoma (ESCC) tissues and paired non-cancer tissues. Using reverse-transcription PCR, western blotting and zymography, we showed that both serine proteases and all three metalloproteinases were highly altered in ESCC. A positive correlation between the expression of FAP- $\alpha$ and DPPIV and the activity of both gelatinases was found. This may indicate that these proteolytic systems are tightly linked to each other and collectively are involved in the process of ECM degradation that facilitates cancer cell invasion and metastasis.
\end{abstract}

\section{Introduction}

Esophageal squamous cell carcinoma (ESCC) is one of the most aggressive gastrointestinal cancers. Despite significant progress in adjuvant therapy, ESCC has the poorest prognosis, and surgical resection still appears to be the only prospect for long-term survival. The main influences on prognosis post-esophagectomy are the depth of tumor invasion and the presence of lymph node metastases, which often accompany even superficial carcinomas. Unfortunately, at the time of diagnosis, more than $50 \%$ of patients have either non-resectable tumors or visible lymph node metastases (1). The invasion

Correspondence to: Dr Katarzyna Augoff, Department of Gastrointestinal and General Surgery, Wroclaw Medical University, Ul. Sklodowskiej-Curie 66, Wroclaw, Poland

E-mail: katarzyna.augoff@umed.wroc.pl

Key words: esophageal squamous cell carcinoma, dipeptidyl peptidase IV, CD26, fibroblast activation protein- $\alpha$, seprase, metalloproteinases and spread of malignant cells is a complex multi-step process that involves degradation and reorganization of the extracellular matrix (ECM) via the activation of different proteolytic systems (2). Proteolysis changes the structure and mechanics of the extracellular scaffold to allow cell migration. It also leads to the release of membrane-anchored cytokines (in particular chemokines) and adhesion molecules, and growth factors stored within the extracellular matrix components (2-4).

Dipeptidyl peptidase IV or CD26 (DPPIV) and fibroblast activation protein- $\alpha$ (FAP- $\alpha$ or F19 cell surface antigen, also known as seprase) are two of a six-member family of serine proteases with post-proline dipeptidyl aminopeptidase activity (5). In their active forms, both enzymes are transmembrane homodimeric glycoprotein complexes with a molecular weight of $\sim 200 \mathrm{kDa}$. The FAP- $\alpha$ and DPPIV genes are located close together on chromosome 2 (q23 and q24.3). They share $52 \%$ amino acid sequence identity, but they differ in their cellular and substrate specificity (5-7). DPPIV is a ubiquitously distributed peptidase that releases a number of biologically active peptides involved in cell growth, migration, invasion, neovascularization or immune system activation (8). These features potentially make DPPIV one of the key players in cancer pathogenesis. FAP- $\alpha$ is a peptidase that cleaves larger proteins and similarly to DPPIV, shows a collagen type I-specific gelatinase activity (9). FAP- $\alpha$ is selectively expressed by myofibroblast-like cells of the tumor stroma, by fibrotic and granulation tissues, and by several types of cancer cells $(6,7)$.

As previously shown, matrix metalloproteinases (MMPs), which are zinc- and calcium-dependent peptidases, are crucial for the initiation and maintenance of ECM degradation. They determine the aggressiveness of cancer cells. Membrane-type metalloproteinase 1 (MT1-MMP/MMP-14) is a member of a membrane-anchored MMP subfamily. It is characterized as an enzyme marker of invadopodia (10). This endopeptidase is synthesized as a 64-kDa pro-enzyme that undergoes furincatalyzed proteolytic cleavage to form an active 54-kDa protein. It not only has the capacity to degrade ECM fibrillar components, proteoglycans or cell surface receptors and cell adhesion molecules, but also acts as a specific initiator of zymogen activation of other MMPs, including metalloproteinase-2 
(MMP-2/gelatinase A) and indirectly metalloproteinase-9 (MMP-9/gelatinase B) (11,12). MMP-2 and MMP-9, two members of the gelatinase subfamily of MMPs, are considered to play a particular role in the early steps of cancer cell invasion and tumor vascularization since they cleave type IV collagen, laminin and elastin, which are the major components of the basement membrane (BM) (3). MMP-2 and MMP-9 are highly homologous proteins, but they differ in their regulation of expression and activation, post-translational modification such as glycosylation, and substrate preferences. Similar to all soluble MMPs, both gelatinases are secreted as inactive pro-enzymes. Their latent forms can be activated in an autocatalytic reaction or through cleavage by serine proteases or other MMPs (3). In addition to their ability to directly degrade extracellular components, MMP-2 and MMP-9 are known to affect cell signaling by releasing the active ectodomain of fibroblast growth factor receptor 1 (FGFR-1) or by activating transforming growth factor $\beta 1$ (TGF- $\beta 1)(13,14)$. MMP-2 and MMP-9 are secreted to the extracellular milieu but both of them localize at the surface of tumor cells. They are able to associate with the MT1-MMP/TIMP-2 complex, $\alpha \mathrm{v} \beta 3$ and $\alpha 3 \beta 1$ integrins, and/or CD44, and thus are involved in local matrix degradation, which enhances the invasive capacity of cancer cells $(3,14,15)$.

As all of the above-mentioned proteases are involved in metastasis and in particular in invadopodia organization, it is crucial to better characterize the role of different membraneassociated proteolytic systems in esophageal cancer growth. To this end, we studied the expression of two serine proteinases, FAP- $\alpha$ and DPPIV, and three metalloproteinases, MMP-2, MMP-9 and MT1-MMP, in 24 primary ESCC tissues paired with non-cancer tissues.

\section{Materials and methods}

Tissue samples and patients. Tissue samples were obtained from 24 patients (19 men and 5 women) with a mean age of 62 years (range, 51-74) who underwent surgery or endoscopic examination of the esophagus in order to diagnose esophageal carcinoma at the Department of Gastrointestinal and General Surgery of Wroclaw Medical University between 2009 and 2012. All of the patients had tumors classified as squamous cell carcinomas. All of the tumors were diagnosed as T3 or T4, N1-N3 and M0-M1 according to the TNM classification. All of the patients had lost $>10 \%$ of their body weight before the diagnosis. They did not have any other serious diseases. All of the cancer samples were paired with non-cancer tissue samples taken from the distal area $(5-10 \mathrm{~cm}$ from macroscopic changes) and confirmed by histological examinations to be R0. In all cases, 3- to 5-mg fragments of each tissue sample were placed in a lysis reagent for total RNA extraction using a Qiagen RNeasy Mini kit. The remainder of each tissue sample was frozen and stored at $-22^{\circ} \mathrm{C}$ until use for western blotting or zymography.

Table I lists the methods used for the study of individual membrane-associated proteases.

The data are expressed as means \pm standard error (SE). The statistical analysis of the data was performed using Student's t-test. The correlation coefficient ( $r$ ) was estimated in order to analyze the correlations between the expression levels of all of
Table I. Methods used for the study of the expression level and the status of activation of the selected membrane-associated proteases.

\begin{tabular}{lccc}
\hline $\begin{array}{l}\text { Membrane-associated } \\
\text { proteases }\end{array}$ & RT-PCR & $\begin{array}{c}\text { Western } \\
\text { blotting }\end{array}$ & $\begin{array}{c}\text { Gelatin } \\
\text { zymography }\end{array}$ \\
\hline FAP- $\alpha$ & Yes & Yes & Yes \\
DPPIV & Yes & Yes & Yes \\
MT1-MMP & & Yes & \\
MMP-2 & & & Yes \\
MMP-9 & & & Yes \\
\hline
\end{tabular}

FAP- $\alpha$, fibroblast activation protein- $\alpha$; DPPIV, dipeptidyl peptidase IV; MMP, matrix metalloproteinase.

the evaluated proteins. Differences were considered significant at $\mathrm{p}<0.05$.

Reverse transcription-polymerase chain reaction (PCR). All the RT-PCR reactions were performed using a one-step RT-PCR kit (Clontech Laboratories, Inc.), according to the manufacturer's instructions. Total RNA $(1 \mu \mathrm{l})$ extracted from each sample was added to a $20 \mu \mathrm{l}$ final reaction volume of RT-PCR mix containing 0.25 pmol of each specific primer. Reverse transcription (RT) was performed for $1 \mathrm{~h}$ at $50^{\circ} \mathrm{C}$ and 5 min at $94^{\circ} \mathrm{C}$. To amplify the FAP- $\alpha$ transcripts, we used the primer pair 5'-gctggagctaagaatcccgttgttcg-3' (sense) and 5'-tgcttggaggatagcttccaatgct-3' (antisense). The FAP- $\alpha$ amplification product length was $544 \mathrm{bp}$. To amplify the DPPIV transcripts, we used the primers 5'-ggaagatggaactgcttagt ggcacg-3' (sense) and 5'-tctcagccetttatcattcacgctgc-3' (antisense). The length of the DPPIV amplification product was $473 \mathrm{bp}$. The PCR conditions were $30 \mathrm{sec}$ at $94^{\circ} \mathrm{C}, 45 \mathrm{sec}$ at $60^{\circ} \mathrm{C}$ and $1 \mathrm{~min}$ at $68^{\circ} \mathrm{C}$ (30 cycles). To detect genomic DNA contamination, RT-free PCR controls were included.

The PCR products were separated and visualized in $1 \%$ agarose gel containing ethidium bromide, digitized and assessed by densitometry using ImageJ software. For the semi-quantitative analysis, the transcripts were related to co-amplified actin DNA, a housekeeping control gene. The actin primers 5'-tacaatgagctgcgtgtggctccccce-3' (sense) and 5'-aatggtgatgacctggccgtcaggc-3' (antisense), yielding a 479-bp amplification product, were used under the same amplification conditions. The pixel density of each individual PCR product was calculated on the basis of the pixel density for the actin transcripts, the values of which were equal to 1 . Results are shown as the ratio of cancer to paired normal tissue.

SDS/PAGE and western blotting. The total protein was extracted from frozen tissues by homogenization $1: 15 \mathrm{w} / \mathrm{v}$ in sample buffer $(0.125 \mathrm{M}$ Tris- $\mathrm{HCl}, \mathrm{pH} 6.8,10 \%$ glycerol, $4 \%$ SDS and 10\% 2-mercaptoethanol). Homogenates were centrifuged for $20 \mathrm{~min}$ at $14,000 \mathrm{x} \mathrm{g}$. All the samples were heated at $95^{\circ} \mathrm{C}$ for $10 \mathrm{~min}$ and electrophoresed in amounts of $10 \mu \mathrm{l} /$ well through a $10 \%$ SDS polyacrylamide gel. After electrophoresis, the samples were transferred at $350 \mathrm{~mA}$ for $1.5 \mathrm{~h}$ to nitrocellulose membranes (Trans-Blot Transfer Medium; 

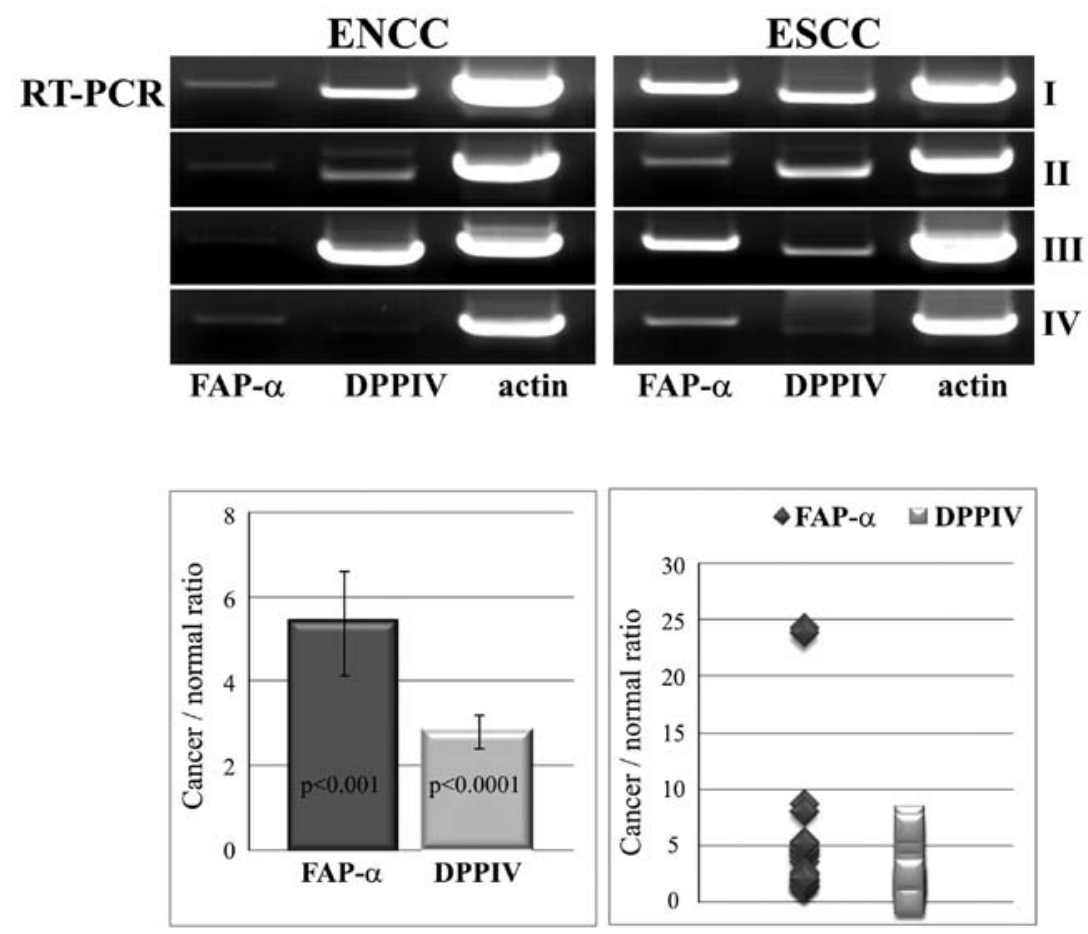

Figure 1. Expression of FAP- $\alpha$ and DPPIV in paired tumor and normal esophageal tissues. (Upper panels) Four representative examples of FAP- $\alpha$ (544-bp amplification product) and DPPIV (473-bp amplification product) mRNA expression as determined using RT-PCR in cancer (ESCC) and non-cancer (ENCC) samples. The expression of the actin transcripts $(479 \mathrm{bp}$ ) is shown as a positive control. I-IV, patient codes. (Bottom panels) Semi-quantitative analysis of the FAP- $\alpha$ and DPPIV PCR products of the control and neoplastic samples. The values are normalized to $\beta$-actin. The results are presented as the ratio of cancer to normal tissue. The bar chart shows mean values and standard error (left). The scatter chart with separate measurements shows the range of calculated values (right). Statistically significant differences between the levels of amplification of both FAP- $\alpha$ and DPPIV cDNA in the cancer and non-cancer tissues were found. ENCC, esophageal non-cancer tissues from areas distant to the tumor. ESCC, esophageal squamous cell carcinoma tissues in the form of paired specimens of malignant tumors. FAP- $\alpha$, fibroblast activation protein- $\alpha$; DPPIV, dipeptidyl peptidase IV.

Bio-Rad Laboratories). The membranes were blocked for $1 \mathrm{~h}$ with 5\% non-fat milk in PBS (pH 7.4) at room temperature and then incubated overnight at $4^{\circ} \mathrm{C}$ with each primary antibody. The antibodies used were: DPP4 antibody-C-terminal region rabbit polyclonal antibody (ARP63319_P050; Aviva Systems Biology); MT-MMP-1 (H-72) rabbit polyclonal antibody (sc-30074); FAP- $\alpha$ (C-19) goat polyclonal antibody (sc-54539) (both from Santa Cruz Biotechnology, Inc., Santa Cruz, CA, USA) and seprase rabbit polyclonal antibody (N1N3) (GTX102732; GeneTex, Inc.). Actin (I-19) goat polyclonal antibody (sc-1616; Santa Cruz Biotechnology, Inc.) was used to ensure equal loading of the proteins.

Immunodetection was performed using HRP-conjugated donkey anti-goat IgGs (sc-2020) or donkey anti-rabbit (sc-2313) (both from Santa Cruz Biotechnology, Inc.) antibodies and a chemiluminescence kit (Pierce Biotechnology, Rockford, IL, USA).

Semi-quantitative digital image analysis was performed with ImageJ software to assess the integral density of particular bands. The integral density of each protein band was normalized to $\beta$-actin (housekeeping protein), the value of which was equal to 1 . Results are shown as the ratio of cancer to paired normal tissue.

Zymography. Tissue extracts were prepared by homogenizing tissue fragments $1: 15 \mathrm{w} / \mathrm{v}$ in a sample buffer consisting of $62.5 \mathrm{mM}$ Tris- $\mathrm{HCl}$ (pH 6.8) with $10 \%$ glycerol, $2 \%$ SDS and $0.05 \%$ bromophenol blue. After a $15-\mathrm{min}$ incubation at room temperature, the homogenates were centrifuged for $15 \mathrm{~min}$ at $14,500 \mathrm{x} \mathrm{g}$. The gelanolytic activity of the MMPs in the supernatants was determined with substrate gel SDS-PAGE zymography. A total of 3 or $7 \mu \mathrm{l}$ of each of the non-reduced samples was loaded in 9\% SDS-polyacrylamide gels copolymerized with gelatin $(2 \mathrm{mg} / \mathrm{ml})$. After semi-native electrophoresis, the enzymes were renaturated by washing SDS out twice in $50 \mathrm{mM}$ Tris- $\mathrm{HCl}(\mathrm{pH} 7.5)$ with $2.5 \%$ Triton X-100 for $30 \mathrm{~min}$ at room temperature. Then the gels were incubated for $20 \mathrm{~h}$ at $37^{\circ} \mathrm{C}$ in $50 \mathrm{mM}$ Tris- $\mathrm{HCl}(\mathrm{pH} \mathrm{7.5)} \mathrm{containing} 150 \mathrm{mM}$ $\mathrm{NaCl}, 10 \mathrm{mM} \mathrm{CaCl}_{2}, 1 \mu \mathrm{M} \mathrm{ZnCl}_{2}$ and $0.05 \%$ Brij-35. An incubation buffer containing $5 \mathrm{mM}$ EDTA was used to identify FAP- $\alpha /$ DPPIV activity. To visualize the proteolytic bands, the gels were stained with $0.12 \%$ Coomassie blue and destained with a solution containing 5\% acetic acid and $10 \%$ ethanol in water. The pixel intensity (in inversion) of the bands, both in their latent and active forms, was determined densitometrically using an Image J gel analyzer showing MMP-2 and MMP-9 activity. The result for each patient was expressed as the ratio of cancer to normal tissue.

\section{Results}

FAP- $\alpha$ and DDPIV are overexpressed in the ESCC tumor tissues. As shown in Fig. 1, FAP- $\alpha$ amplification products corresponding to the predicted ones were generated from all the tumor samples and notably, also from all the non-cancer esophageal tissue samples. For the semi-quantitative analysis, 


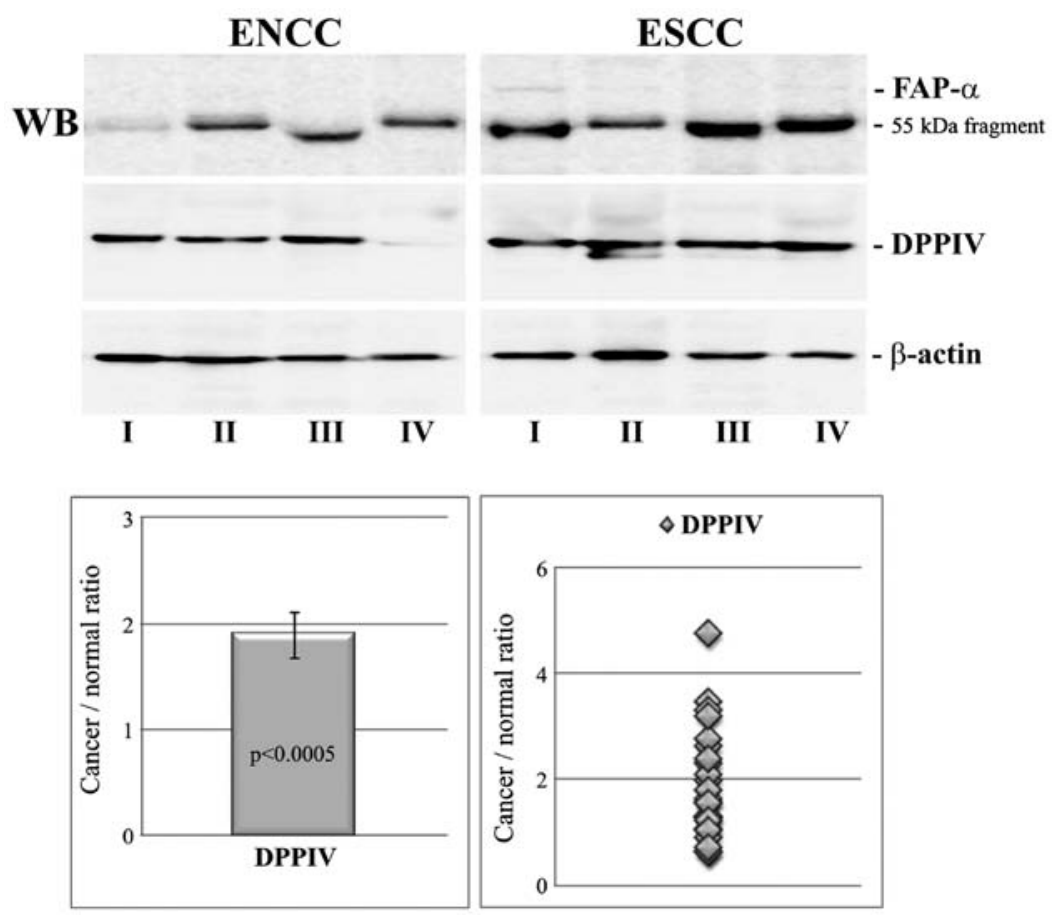

Figure 2. FAP- $\alpha$ and DPPIV protein levels in tumor and normal esophageal tissues. (Upper panels) Examples of FAP- $\alpha$ western blot immunostaining of four paired non-cancer (ENCC) and cancer (ESCC) samples. The bands indicate a 97-kDa full-length monomeric form in three cancer tissues and a 55-kDa proteolytic fragment in all of the non-cancer and cancer samples. Immunodetection of $\beta$-actin, a 43-kDa protein, was used for normalization. I-IV, patient codes. (Bottom panels) Semi-quantitative analysis of DPPIV in the control and neoplastic samples. Post-normalization data are presented as the ratio of cancer to normal tissue. The bar chart shows the mean values and standard error (left). The scatter chart with separate measurements shows the range of calculated values (right). Statistically significant differences were found between the level of DPPIV protein in the cancerous and non-cancerous tissues. FAP- $\alpha$, fibroblast activation protein- $\alpha$; DPPIV, dipeptidyl peptidase IV; ESCC, esophageal squamous cell carcinoma.
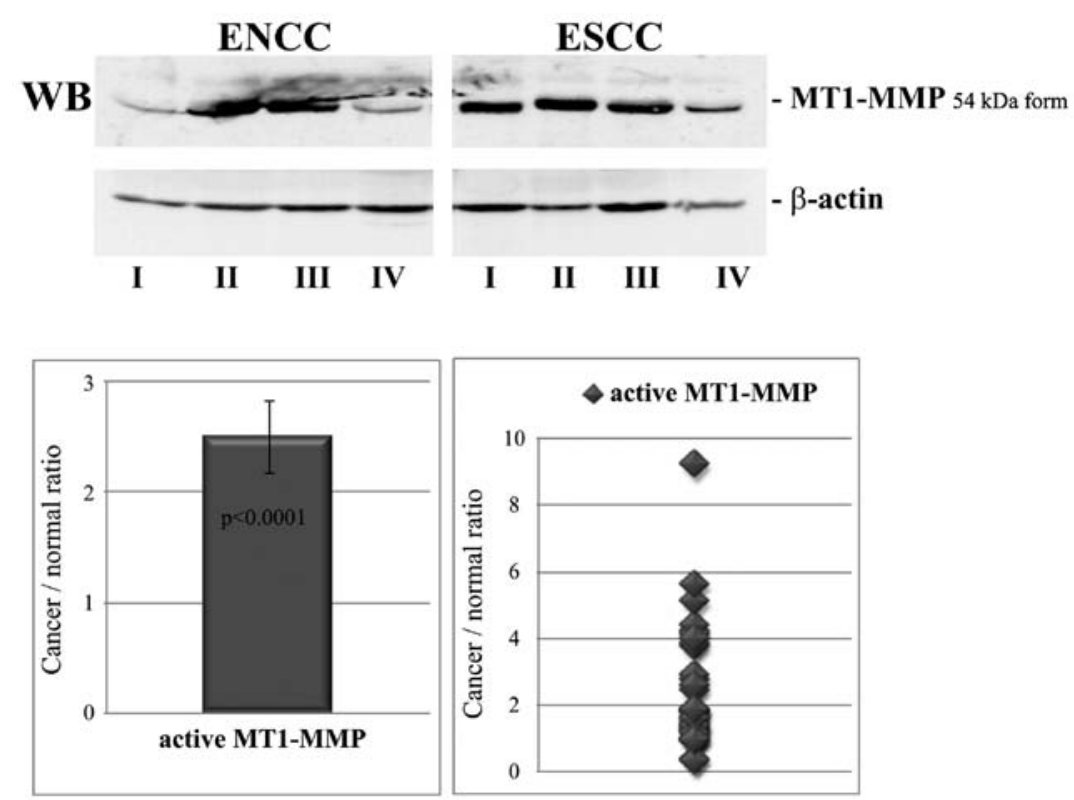

Figure 3. Levels of the MT1-MMP active form in cancer and non-cancer esophageal tissues. (Upper panels) Representative MT1-MMP western blotting of four paired non-cancer (ENCC) and cancer (ESCC) samples. The bands indicate the 54-kDa active form. Immunodetection of the 43-kDa protein, $\beta$-actin, was used for normalization. I-IV, patient codes. (Bottom panels) Ssemi-quantitative analysis of the activated MT1-MMP form in the control and neoplastic samples. Post-normalization data are presented as the ratio of cancer to normal tissue. The bar chart shows the mean values and standard error (left). The scatter chart with separate measurements shows the range of calculated values (right). Statistically significant differences between the levels of MT1-MMP activation in cancerous and non-cancerous tissues. MMP, matrix metalloproteinase; ESCC, esophageal squamous cell carcinoma.

we co-amplified actin transcripts in all of the samples under the same RT-PCR conditions. We found a much lower ampli- fication of FAP- $\alpha$ transcripts in the control in comparison with the cancer tissue samples. The differences between the 

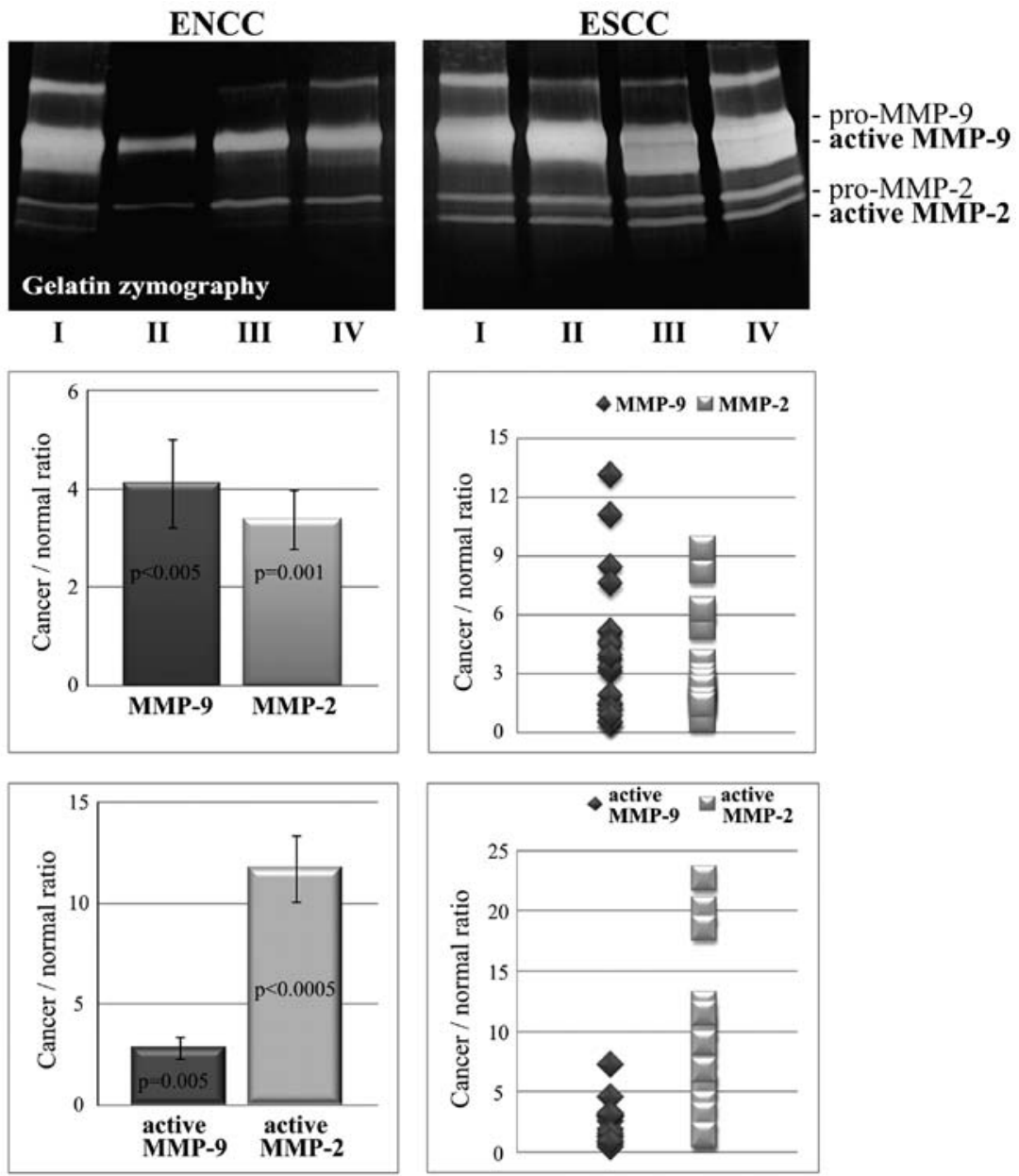

Figure 4. Gelatinolytic activity in cancer and non-cancer esophageal tissues. (Upper panels) Representative gelatin zymogram showing the pro- and active forms of MMP-2 (lower bands) and MMP-9 (upper bands) in four paired control (ENCC) and cancer (ESCC) tissues. I-IV, patient codes. (Middle panels) Semi-quantitative analysis of the total MMP-9 and MMP-2 protein levels in the control and neoplastic samples. The normalized data are presented as the ratio for cancer to normal tissue. The bar chart shows mean values and standard error (left). The scatter chart with separate measurements shows the range of calculated values (right). Significantly increased levels of total gelatinase synthesis were found in the cancer tissue samples. (Bottom panels) Semi-quantitative analysis of the levels of MMP-9 and MMP-2 active forms in the control and neoplastic samples. The final data are presented as the ratios of cancer to normal tissues. Statistically significant differences were found between the ratios of activation of MMP-2 and MMP-9 in the tumor and normal tissues. The bar chart presents means and standard errors (left). The scatter chart shows the range of separate measurements (right). MMP, matrix metalloproteinase; ESCC, esophageal squamous cell carcinoma.

groups were statistically significant $(\mathrm{p}<0.001)$. The mean ratio of cancer to normal tissue was calculated to be $5.387 \pm 1.23$ (range, 1.229-24.258).

DPPIV transcripts were found in all the analyzed cancer and non-cancer tissue samples, with significantly higher expression of DPPIV in the pathological samples than that in the control samples $(\mathrm{p}<0.0001)$. However, the ratio of cancer to normal tissue for DPPIV, calculated as 2.811 \pm 0.41 (range, 0.162-7.139), was not as high as for FAP- $\alpha$ (Fig. 1). Upregulation of DPPIV was found to positively correlate with the FAP- $\alpha$ expression ratio $(r=0.58, p=0.002)$. No genomic DNA transcripts for FAP- $\alpha$ or DPPIV were found in the RT-free PCR controls. The FAP- $\alpha$ and DPPIV transcripts were verified by sequencing.

We used the western blotting technique to determine the protease expression at the protein level. The results were normalized to $\beta$-actin and are shown in Fig. 2 . The ratio of cancer to normal tissue for DPPIV was in the range of $0.63-4.75$ with a mean value of $1.89 \pm 0.21$. The increased level of DPPIV in ESCC was statistically significant $(\mathrm{p}<0.0005)$ and was correlated positively with DPPIV expression at the mRNA level $(\mathrm{r}=0.725, \mathrm{p}=0.023)$.

The weak signal in immunoblots in the case of FAP- $\alpha$ did not allow densitometric analysis to be performed. Of the 24 cancer tissue samples, only three were unequivocally immunoreactive for full-length protein. All three FAP- $\alpha$-positive cases are shown in Fig. 2 . In the remaining cases, only proteolytic fragments of $\sim 55 \mathrm{kDa}$ were observed. Gelatin zymography was also not sensitive enough to detect serine protease activity in most of the tissue extracts.

MT1-MMP, MMP-2 and MMP-9 are upregulated in ESCC tumor tissues. The levels of the active 54-kDa form of MT1-MMP were evaluated by western blot analysis after normalization to $\beta$-actin. The results are shown in Fig. 3. The ratio of cancer to normal tissue for activated MT1-MMP was in the range of $0.33-9.25$ with a mean value of $2.5 \pm 0.33$. The increased level of the MT1-MMP active form in ESCC was statistically significant $(\mathrm{p}<0.0001)$. 
As shown in Fig. 4, the values for the ratio of cancer to normal tissue calculated on the basis of zymogram gels for total (active and latent form) MMP-2 (3.38 \pm 0.6 ; range, 0.68-9.4) and total (active and latent form) MMP-9 (4.11 \pm 0.89 ; range, $0.49-13.12$ ) were comparable. The levels of the two gelatinases in the cancer tissues were significantly higher than these levels in the non-cancer tissues $(\mathrm{p}=0.001$ and $\mathrm{p}<0.005$, respectively). The relative increase in activity of MMP-2 was not significantly different than the relative increase in activity of MMP-9. However, the upregulation of the activities of the two gelatinases was found to be positively correlated $(\mathrm{r}=0.819$, $\mathrm{p}=0.001)$. The ratio of cancer to normal tissue for the active MMP-2 form was $11.75 \pm 1.65$ (range, 5.57-22.68) but only $2.85 \pm 0.52$ (range, 0.46-7.28) for the active form of MMP-9. In ESCC, the increase in gelatinase activity was statistically significant for MMP-2 ( $\mathrm{p}<0.00005)$ and MMP-9 $(\mathrm{p}=0.005)$. A positive correlation was found between the ratios calculated for the active form of gelatinase A and MT1-MMP (correlation, $\mathrm{r}=0.93 ; \mathrm{p}=0.007)$. Changes in MMP-2 and MMP-9 activity were found to positively correlate with the FAP- $\alpha$ and DPPIV expression levels (correlation, $r=0.9 ; p<0.05$ ).

\section{Discussion}

Cancer invasion and the formation of metastases are based on the ability of cells to degrade and remodel the extracellular scaffold. Deregulation of protease expression and activity has been reported in a large number of epithelial cancers, including tumors of the esophagus $(16,17)$. The participation of proteases in tumor development and progression not only involves their direct participation in extracellular matrix degradation but also in maintaining communication between tumor cells and the microenvironment.

FAP- $\alpha$ and DPPIV are cell surface glycoproteins that act as post-proline-specific peptidases. They are highly expressed in areas of active tissue remodeling. Since many biologically active peptides contain a conserved proline residue as a proteolytic-processed regulatory site, FAP- $\alpha$ and DPPIV may be recognized as important regulators of extracellular signaling. In addition to their typical dipeptidyl peptidase activity, FAP- $\alpha$ and DPPIV possess collagenolytic activity. Due to their cellular location, they are known to be proteases associated with sites of cell adherence to the ECM $(6,9)$.

The variety of functions included in the range of DPPIV activity indicates that the mechanisms by which DPPIV affects the behavior of tumor cells can vary in different types of cancer, even resulting in the opposite roles. It was shown that DPPIV overexpression in ovarian cancer cells induces downregulation of MT1-MMP and MMP-2 and upregulates tissue inhibitors of metalloproteases (TIMPs), leading to the suppression of the invasive potential of the cells $(18,19)$. Several studies also indicated that the loss of expression of DPPIV was linked with malignant transformation and tumor progression. On the other hand, increased expression of DPPIV was reported in prostate and thyroid carcinomas $(20,21)$.

We found that the average 3 -fold increase in DPPIV gene expression and an 2 -fold protein level increase in ESCC over the controls was positively correlated with the intensity of MMP-2 and MMP-9 activity. DPPIV was present in all of the normal and cancer tissues. Goscinski et al investigated the level of DPPIV expression in ESCC via immunohistochemistry (22). They observed positive staining for DPPIV in the majority of cancer tissues. They did not find DPPIV immunoreactivity in the normal esophageal epithelium. Mentlein et al showed that various types of cells in healthy tissue, such as certain fibroblasts, endothelial cells or activated T or NK cells, express high levels of DPPIV (23). Therefore, the presence of DPPIV in non-cancer tissues was not surprising.

In the present study, using RT-PCR, we found an average 5 -fold lower level of FAP- $\alpha$ expression in the normal noncancer tissues than that in the cancer cases, but all of the studied controls were FAP- $\alpha$ mRNA-positive. It is accepted that FAP- $\alpha$ is not expressed by cells of mature somatic tissues, except by the myofibroblasts of granulation or fibrotic tissues or myofibroblast-like cells of tumor stroma (7). In esophageal cancers, intense FAP- $\alpha$ immunostaining has already been observed (24). The positive immune reaction was also found in stroma adjacent to cancer foci and even in areas adjacent to dysplastic changes. However, FAP- $\alpha$ was not detected in the microscopic normal epithelium of the esophagus. In fact, immunohistochemistry is much less sensitive than molecular techniques. Therefore, the open question is whether or not the presence of FAP- $\alpha$ in the surgical margin may be evidence supporting the view that the stromal changes extend much farther into the surrounding tumor tissues than is usually considered. Alterations in the stromal environment are known to precede cancer invasion and are closely associated with myofibroblast-like cells. One of the major markers of myofibroblasts is $\alpha$-smooth muscle actin ( $\alpha$-SMA) (25). We estimated the expression of $\alpha$-SMA in ESCC and paired noncancer tissues (data not shown) but these data did not correlate with the FAP- $\alpha$ expression level.

MT1-MMP is a key mediator in ECM proteolysis $(4,10)$. MT1-MMP was the first membrane MMP discovered on the surface of invasive cells. Various authors demonstrated that MT1-MMP is overexpressed in different cancer cell lines and tumor tissue types $(26,27)$. It was also shown that specific MT1-MMP downregulation suppresses the migration and invasion of cancer cells (28). In the present study, we found a much higher level of the active MT1-MMP form in cancers relative to paired non-cancer esophageal tissues. The correlation between the level of the MT1-MMP active form and the MMP-2 activity found in the present study shows a tight connection between the two enzymes and concurs with the data in previous reports describing the role of MT1-MMP as an MMP-2 activator (29). A lack of correlation between activated MT1-MMP and the remaining proteases may suggest that MT1-MMP is not directly involved in the mechanisms of their expression and/or activation in ESCC.

Upregulation of MMP-2 and MMP-9 expression was observed in almost all human cancers and was found to significantly correlate with the histological grade of the cancer, with metastases and with patient survival $(17,30)$. Therefore, the active contribution of both gelatinases to cancer progression is evident. In the present study, we also found the enhanced synthesis of both MMP-2 and MMP-9 in cancer tissues. The most significant result of our analysis was the extremely high increase in MMP-2 activity in ESCC tissues. It was relatively higher than that for MMP-9. Despite the differences 
in activation, the ratio of cancer to normal tissues of the two active gelatinases correlated positively with the expression levels of both serine proteases. This suggests that MMP-2, MMP-9, FAP- $\alpha$ and DPPIV may function coordinately to regulate the behavior of cancer cells.

In summary, this study demonstrated that different membrane-associated proteolytic systems, including transmembrane proteases, such as FAP- $\alpha$, DPPIV and MT1-MMP, and the membrane-attached matrix metalloproteinases, such as MMP-2 and MMP-9, are highly altered in ESCC. The positive correlation between the expression of serine proteases and the activity of both gelatinases indicates that all these proteolytic systems may be tightly linked to each other and may collectively improve focal ECM degradation, which facilitates cancer cell invasion and metastasis.

\section{References}

1. Ilson DH: Oesophageal cancer: new developments in systemic therapy. Cancer Treat Rev 29: 525-532, 2003.

2. Ellis V and Murphy G: Cellular strategies for proteolytic targeting during migration and invasion. FEBS Lett 506: 1-5, 2001.

3. Björklund $M$ and Koivunen E: Gelatinase-mediated migration and invasion of cancer cells. Biochim Biophys Acta 1755: 37-69, 2005.

4. Poincloux R, Lizárraga F and Chavrier P: Matrix invasion by tumour cells: a focus on MT1-MMP trafficking to invadopodia. J Cell Sci 122: 3015-3024, 2009.

5. Chen WT and Kelly T: Seprase complexes in cellular invasiveness. Cancer Metastasis Rev 22: 259-269, 2003.

6. Kelly T: Fibroblast activation protein- $\alpha$ and dipeptidyl peptidase IV (CD26): cell-surface proteases that activate cell signaling and are potential targets for cancer therapy. Drug Resist Updat 8: 51-58, 2005.

7. O'Brien P and O'Connor BF: Seprase: an overview of an important matrix serine protease. Biochim Biophys Acta 1784: $1130-1145,2008$

8. Boonacker $E$ and Van Noorden CJ: The multifunctional or moonlighting protein CD26/DPPIV. Eur J Cell Biol 82: 53-73, 2003.

9. Baum O, Reutter W and Bermpohl F: Structure-function relationship of DPP IV: insights into its dimerisation and gelatinase activity. Adv Exp Med Biol 524: 19-27, 2003.

10. Itoh Y: MT1-MMP: a key regulator of cell migration in tissue. IUBMB Life 58: 589-596, 2006.

11. Murphy G, Stanton H, Cowell S, et al: Mechanisms for pro matrix metalloproteinase activation. APMIS 107: 38-44, 1999.

12. Toth M, Chvyrkova I, Bernardo MM, et al: Pro-MMP-9 activation by the MT1-MMP/MMP-2 axis and MMP-3: role of TIMP-2 and plasma membranes. Biochem Biophys Res Commun 308: 386-395, 2003

13. Levi E, Fridman R, Miao HQ, et al: Matrix metalloproteinase 2 releases active soluble ectodomain of fibroblast growth factor receptor 1. Proc Natl Acad Sci USA 93: 7069-7074, 1996.

14. Egeblad M and Werb Z: New functions for the matrix metalloproteinases in cancer progression. Nat Rev Cancer 2: 161-174, 2002 .
15. Monsky WL, Kelly T, Lin CY, et al: Binding and localization of $\mathrm{M}_{\mathrm{r}}$ 72,000 matrix metalloproteinase at cell surface invadopodia. Cancer Res 53: 3159-3164, 1993.

16. Etoh T, Inoue H, Yoshikawa Y, et al: Increased expression of collagenase-3 (MMP-13) and MT1-MMP in oesophageal cancer is related to cancer aggressiveness. Gut 47: 50-56, 2000.

17. Roh MR, Zheng Z,Kim HS, et al: Differential expression patterns of MMPs and their role in the invasion of epithelial premalignant tumors and invasive cutaneous squamous cell carcinoma. Exp Mol Pathol 92: 236-242, 2012.

18. Kajiyama H, Kikkawa F, Khin E, et al: Dipeptidyl peptidase IV overexpression induces up-regulation of E-cadherin and tissue inhibitors of matrix metalloproteinases, resulting in decreased invasive potential in ovarian carcinoma cells. Cancer Res 63: 2278-2283, 2003.

19. Kikkawa F, Kajiyama H, Shibata K, et al: Dipeptidyl peptidase IV in tumor progression. Biochim Biophys Acta 1751: 45-51, 2005.

20. Kotani T, Aratake Y, Ogata Y, et al: Expression of dipeptidyl aminopeptidase IV activity in thyroid carcinoma. Cancer Lett 57: 203-208, 1991.

21. Wilson MJ, Ruhland AR, Quast BJ, et al: Dipeptidylpeptidase IV activities are elevated in prostate cancers and adjacent benign hyperplastic glands. J Androl 21: 220-226, 2000.

22. Goscinski MA, Suo ZH, Nesland JM, et al: Dipeptidyl peptidase IV expression in cancer and stromal cells of human esophageal squamous cell carcinomas, adenocarcinomas and squamous cell carcinoma cell lines. APMIS 116: 823-831, 2008.

23. Mentlein R, Hattermann K, Hemion C, et al: Expression and role of the cell surface protease seprase/fibroblast activation protein- $\alpha$ (FAP- $\alpha$ ) in astroglial tumors. Biol Chem 392: 199-207, 2011.

24. Goscinski MA, Suo ZH, Nesland JM, et al: Seprase, dipeptidyl peptidase IV and urokinase-type plasminogen activator expression in dysplasia and invasive squamous cell carcinoma of the esophagus. A study of 229 cases from Anyang Tumor Hospital, Henan Province, China. Oncology 75: 49-59, 2008.

25. Yamashita M, Ogawa T, Zhang X, et al: Role of stromal myofibroblasts in invasive breast cancer: stromal expression of alpha-smooth muscle actin correlates with worse clinical outcome. Breast Cancer 19: 170-176, 2012.

26. Jiang WG, Davies G, Martin TA, et al: Expression of membrane type-1 matrix metalloproteinase, MT1-MMP in human breast cancer and its impact on invasiveness of breast cancer cells. Int $\mathbf{J}$ Mol Med 17: 583-590, 2006.

27. Adley BP, Gleason KJ, Yang XJ and Stack MS: Expression of membrane type 1 matrix metalloproteinase (MMP-14) in epithelial ovarian cancer: high level expression in clear cell carcinoma. Gynecol Oncol 112: 319-324, 2009.

28. Ueda J, Kajita M, Suenaga N, et al: Sequence-specific silencing of MT1-MMP expression suppresses tumor cell migration and invasion: importance of MT1-MMP as a therapeutic target for invasive tumors. Oncogene 22: 8716-8722, 2003.

29. Nakamura $H$, Ueno $H$, Yamashita $K$, et al: Enhanced production and activation of progelatinase A mediated by membrane-type 1 matrix metalloproteinase in human papillary thyroid carcinomas. Cancer Res 59: 467-4573, 1999.

30. Rosenthal EL and Matrisian LM: Matrix metalloproteases in head and neck cancer. Head Neck 28: 639-648, 2006. 